\title{
Hormonal and Metabolic Disorders of Human Immunodeficiency Virus Infection and Substance Abuse
}

\author{
Jag H. Khalsa, Frank Vocci and ${ }^{1}$ Adrian Dobs \\ National Institute on Drug Abuse, National Institutes of Health, Bethesda, Maryland \\ ${ }^{1}$ Johns Hopkins University School of Medicine, Baltimore, Maryland
}

\begin{abstract}
There are an estimated 200 million users of an illicit drug in the world today. In addition, an estimated 40 million people are infected with the human immunodeficiency virus (HIV) and an estimated 180 million people are infected with the hepatitis C virus (HCV). Both the use of an illicit drug and the co-occurrence of infections are associated with a multitude of medical and health consequences including hormonal and metabolic disorders. Thus, the National Institute on Drug Abuse (NIDA), a part of the National Institutes of Health (NIH) hosted a workshop on hormonal and metabolic disorders of HIV among substance abusers. A number of clinicians and scientists participated and discussed a wide range of issues concerning hormones, nutrition and metabolic complications in HIV and substance abuse. Their observations and the recommendations they made for future research are presented in these proceedings. The readers are encouraged to contact the NIH staff $(\mathrm{JK}, \mathrm{FV})$ for technical guidance and programmatic priorities on the subject and directly contact the individual authors for collaborations.
\end{abstract}

Key words: Hormonal and metabolic disorders, viral infection, substance abuse, immunodeficiency

\section{INTRODUCTION}

Substance abuse is one of the major problems in the world today. The World Health Organization estimates that there are an estimated 200 million users of an illicit drug ${ }^{[1,2]}$ in the world. According to the 2004 National Survey on Drug Use and Health, approximately 110 million Americans 12 years of age and older (46\%) used at least one illicit drug in their lifetime ${ }^{[3]}$. In addition, in the world, an estimated 40 million people are living with human immunodeficiency virus/acquired immune deficiency syndrome (HIV/AIDS) and an estimated 180 million people are living with hepatitis $\mathrm{C}$ virus (HCV) infection. In the United States, an estimated 1 million people are infected with HIV and 4 million people are infected with $\mathrm{HCV}^{[4]}$. Both infections are prevalent among substance abusers. For example, CDC estimated that that injection drug use (IDU) directly and indirectly accounts for more than one-third (36\%) of AIDS cases; of the 42, 156 new cases of AIDS reported in 2000, 11,635 (28\%) were IDU-associated; about 50 percent to 85 percent of injection drug users are coinfected with HIV as well as with HCV. Both drug abuse and $\mathrm{HIV} / \mathrm{HCV}$ infections are associated with serious medical/health consequences that may include immunological, cardiovascular, hepatic, hormonal and metabolic complications.
With these facts in mind, in August 2004 the National Institute on Drug Abuse, a part of the National Institutes of Health, U.S. Department of Health and Human Services, organized a symposium on the role of hormones in drug abuse and the dual infections of HIV and HCV. Outstanding clinicians and researchers discussed a wide range of issues concerning hormones, nutrition and metabolic complications in HIV- and HIV/HCV-infected substance abusers. Participants also made several recommendations for future research.

Dobs $^{[5]}$ reported on gonadal and hormonal abnormalities among HIV-infected drug abusers in the City of Baltimore, Maryland. She stated that the use of opiates and cocaine was associated with effects on adrenal and gonadal function; opiate use suppressed the hypothalamic-pituitary-adrenal (HPA) axis, whereas cocaine use activated the HPA axis. Opiates also cause gonadal dysfunction in both men and women. During withdrawal from opiates and cocaine, the HPA axis was activated, which might reinforce relapse behavior. Dobs further reviewed these hormonal effects and explored the potential consequences, including the effects on mood, cognition and cardiovascular risk. She suggested that modification of the drug-induced hormonal dysfunction might represent a treatment strategy for drug rehabilitation.

Kumar $^{[6]}$ described neuroendocrine abnormalities in HIV-infected drug abusers. He stated that since IDU was an important risk factor for becoming infected with 
HIV, it is possible that substance use also might be be associated with endocrine dysfunction. Kumar described his group's study of HPA axis activity in which they investigated cortisol response to the cold pressor challenge in injection drug users with and without HIV infection. He showed that the pattern of cortisol response depended on the person's serostatus. Cortisol levels peaked later and recovered at a slower rate in HIV-positive injection drug users than in HIVnegative injection drug users, thereby suggesting that IDU and HIV infection were associated with dysregulation of HPA axis activity.

Mantzoros $^{[7]}$ discussed the role of leptin and adiponectin, two newly discovered adipose tissuederived hormones with important roles in energy homeostasis and insulin resistance, in the HIVassociated metabolic syndrome. He also reviewed their interrelationships with the manifestations of HIVassociated fat redistribution. Mantzoros described an association of the HIV metabolic syndrome with a state of reduced insulin action due to adiponectin deficiency observed concurrently with an increased lipogenesis state due to enhanced SREBP-1c expression and/or other mechanisms. This is accompanied by leptin deficiency in lipoatrophic subjects and possibly by a leptin resistance state in lipohypertrophic patients. He suggested that adiponectin and/or leptin therapy in a manner similar to other leptin deficiency states might assist in the future management of such patients after taking into account the fat redistribution phenotype and the pattern of metabolic changes observed.

Kotler $^{[8]}$ reported on the effects of growth hormone (GH) therapy in HIV-infection. He showed that recombinant human growth hormone (rhGH) might play a role in the management of visceral fat accumulation associated with HIV infection. Kotler also showed that the effects of various doses of rhGH on trunk fat fit a dose-response characteristic. However, three major issues remain unresolved: (a) definitions that are clinically applicable methods of diagnosing visceral fat accumulation, (b) monitoring its response during treatment and (c) the appropriate maintenance dose for long-term therapy. Although it is clear that one is not treating just a body compartment but rather a factor related to cardiovascular risk, an understanding of how to incorporate this information into a management scheme is elusive at present. In the event that therapy is chosen, the optimal induction dose still needs definition, as does the overall question of whether induction is needed in the face of long-term maintenance therapy.

Grunfeld $^{[9]}$ reported on the metabolic abnormalities associated with the use of protease inhibitors (PIs) and nonnucleoside reverse transcriptase inhibitors (NNRTIs). He reported that the use of PIs and NNRTIs was associated with multiple abnormalities in glucose and lipid metabolism such as insulin resistance, increased triglycerides and increased levels of low- density lipoprotein cholesterol. Grunfeld stated that the metabolic disturbances might be due to a combination of factors, including the direct effect of medications, restoration to health and HIV disease as well as individual genetic predisposition. Of the available antiretroviral medications, indinavir had been associated with causing the most insulin resistance and ritonavir with causing the most hypertriglyceridemia.

Balasubramanyam ${ }^{[10]}$ presented new evidence from his group's metabolic and molecular studies to explain the underlying pathophysiology of adipocyte defects and dyslipidemia in HIV lipodystrophy. He argued that despite a burgeoning mass of descriptive information regarding the epidemiology, clinical features, body composition changes, hormonal alterations and dyslipidemic patterns in patients with HIV lipodystrophy syndrome (HLS), the specific biochemical pathways that are dysregulated in the condition and the molecular mechanisms that lead to their dysfunction remain relatively unexplored. Balasubramanyam reviewed a number of studies that detailed the metabolic basis of the dyslipidemiaspecifically, the hypertriglyceridemia-that is the serologic hallmark of HLS and presented new data relevant to the mechanisms of dyslipidemia in the postprandial state. He also described their preliminary experiments showing that in addition to the well-known effects of highly active antiretroviral drugs, the functional disruption of adipocytes and preadipocytes by factors intrinsic to HIV-infected immunocytes might play a role in the pathogenesis of HLS.

Baum $^{[11]}$ discussed the risk factors of coronary heart disease (CHD) and metabolic syndrome in HIVinfected drug abusers. She reported that the frequency of CHD was increasing among HIV-positive persons and that this phenomenon might be related to HIV disease itself, the use of antiretroviral medications and the increased length of survival-or the synergism between or among any of these factors. Baum showed that the 10-year CHD risk among the HIV-positive drug abusers was approximately five times higher in infected people than in noninfected people. The risk also was higher in men than in women, despite a significantly higher incidence of abdominal obesity and lower highdensity lipoprotein (HDL) levels in women. The rate of metabolic syndrome among female HIV-positive drug abusers was significantly higher than in men. Participants with metabolic syndrome had a significantly higher 10-year CHD risk and higher mean body mass index than those without the syndrome. Participants also had higher viral loads, suggesting that their use of illicit drugs had an influence on either adherence to or effectiveness of antiretroviral medication. Increased viral load was significantly associated with metabolic syndrome, high fasting glucose and low HDL levels. Heavy use of cigarettes and crack/cocaine was inversely associated with obesity, whereas marijuana use was associated with 
increased central obesity. The significant association of higher viral load with CHD risk indicated that controlling viral load might be important in reducing CHD risk in HIV-infected drug abusers.

Forrester $^{[12]}$ reviewed the published literature on the nutritional status of drug abusers with and without HIV, with emphasis on dietary energy and macronutrient intake. She stated that many studies found that drug abusers had nutritional deficits, including weight deficits and that the most plausible explanation for these deficits was dietary insufficiency. However, studies using objective measures of the dietary intake of drug abusers had failed to provide evidence of dietary insufficiency. Other mechanisms rarely had been examined. Forrester recommended additional studies to look for other unexplored mechanisms that could explain the nutritional deficiencies found in HIV-infected drug abusers.

Grinspoon $^{[13]}$ discussed the mechanisms of insulin resistance among HIV-infected patients with problems of fat distribution and recent strategies used for this population. The mechanism of insulin resistance among HIV-infected patients with fat redistribution might be related to the direct effects of antiretroviral agents on glucose transport, changes in body composition and substrate flux, including increased intramyocellular lipids or altered levels of adipocytokines. He pointed out that recent strategies had focused on the use of insulin-sensitizing agents and that metformin had been shown to improve insulin sensitivity and to decrease visceral fat. In contrast, among insulin-resistant patients, rosiglitazone had been shown to increase subcutaneous adipogenesis and to improve adiponectin and insulin sensitivity.

$\mathrm{Hruz}^{[14]}$ discussed the molecular mechanisms for altered glucose homeostasis in HIV infection. He stated that a complete understanding of the molecular mechanisms leading to HIV-associated insulin resistance remains elusive and that there are complex interrelationships among genetic predisposition, disease-related body changes and multidrug therapy that might contribute to alterations in glucose homeostasis. These abnormalities could be differentiated between acute and reversible changes directly induced by highly active antiretroviral therapy (HAART), medications and more chronic and less reversible changes due to the development of lipodystrophy and hyperlipidemia. Pathways such as changes in adipokine secretion, insulin signaling, lipid homeostasis and disease-related increases in inflammatory mediators also could be implicated. The insulin-responsive facilitative glucose transporter GLUT4 was the first molecule to be identified as a direct target of HIV PIs. Efforts to elucidate the mechanisms directly responsible for the evolution of insulin resistance during $\mathrm{HIV}$ infection and therapy would be greatly assisted by the further identification and characterization of direct molecular targets amenable to pharmacologic therapy and/or the development of new antiretroviral agents that do not adversely affect these target proteins.

Finally, participants recommended that, wherever feasible, the following basic and clinical research be conducted to study the impact and role of illicit drug use in hormonal and metabolic complications of dual infections of HIV and hepatitis C.

In summary, both substance abuse and cooccurrence of infections such as HIV and HCV are associated with serious medical and health consequences including hormonal and metabolic (including nutritional) disorders. Drug abuse alone or in the presence of co-occurring HIV infection was associated with gonadal dysfunction via modulation of the activity of HPA axis. Research suggests that the newly discovered adipose tissue-derived hormones, adiponectin and/or leptin, may be playing an important role in energy homeostasis and insulin resistance in the HIV-associated metabolic syndrome. The recombinant human growth hormone (rhGH) might play a role in the management of visceral fat accumulation associated with HIV infection. The metabolic complications involving glucose (insulin resistance) and lipid metabolism (hypertriglyceridemia), associated with the use of antiretroviral PIs and NNRTIs, might be due to direct effect of medications, restoration to health, HIV disease, underlying molecular mechanisms involving adipocytes and preadipocytes and individual genetic predisposition. Other complications of metabolic dysregulation such as coronary heart disease are also possible in HIV-infected drug abusers. But participants also indicated that there is paucity of data on the extent, nature and underlying pathophysiology of hormonal and metabolic complications and various intervention strategies that could be used in the treatment of these complications and recommended research on these issues. These are summarized in these proceedings.

\section{RECOMMENDATIONS FOR FUTURE RESEARCH}

* Determine the safety of the long-term use of testosterone in HIV-infected men who abuse illicit drugs and study the long-term significance of endocrine abnormalities on end-organs such as the cardiovascular and other systems.

* Study the temporal relationship between drug abuse and endocrine abnormalities, the interrelationship between the HPA axis and the hypothalamic-pituitary-gonadal axis and the effect of duration and extent of drug use on endocrine disorders.

* Conduct clinical trials of testosterone supplementation on the progression of atherosclerosis and clinically relevant outcomes in $\mathrm{HIV}$-associated metabolic disorders in drug abusers. 
* Study molecular and signaling mechanisms in the regulation of currently available stem cell lines; these have enormous implications for drug discovery.

* Study the relationship between neuroendocrine regulation and cognitive deficits in HIV infection.

* Study the role of glucocorticoids in neurodegeneration in determining the effects of drug use versus HIV infection on neuroendocrine abnormalities.

* Study the residual neuroendocrine and cytokine abnormalities of antiretroviral intervention.

* Study the pathogenetic mechanisms of insulin resistance in the $\mathrm{HIV}$-infected population; determine the optimal use of insulin sensitizing agents in the lipodystrophy population; and determine the role of adipocytokines in HIV infection.

* Identify and characterize the direct molecular targets of antiretroviral drugs capable of contributing to impaired insulin action during HAART; screen antiretroviral agents in development for their ability to alter the functional activity of drug targets known to contribute to insulin resistance.

* Study the etiology and pathogenesis of glucoregulation and insulin resistance in HIV infection and drug abuse; support prospective clinical trials on pharmacotherapy for insulin resistance.

* Study the effects of myostatin on lipodystrophy, the effects of antimyostatin antibodies on HIVassociated muscle wasting, the effects of myostatin blockers on leptin levels and the effects of low organ weights on metabolism in HIV-positive individuals on HAART?

* Study the molecular mechanisms of adipocyte function in conditions such as chronic inflammation, fatty liver and steatosis in HIV infection and the impact of illicit drugs on adipocyte function.

* Study the role of illicit drugs on adipokines in the etiology of the HAART-induced metabolic syndrome.

* Study the biochemical and molecular mechanisms, including the role of genetic and other host factors, in the pathogenesis of lipodystrophy; study $\mathrm{HIV} / \mathrm{HCV}$ interactions in "lipodystrophy syndrome," particularly with respect to hepatic steatosis; determine the optimal treatment, particularly studies of novel therapies (e.g., leptin), as well as new combinations of existing therapies (e.g., GH plus a thiazolidinedione) for lipodystrophy.

* Establish an international consortium to study the rare types of inherited lipodystrophies to discover additional lipodystrophy genes, develop knock-out or transgenic nonhuman animal models of various human lipodystrophy genes to understand the underlying molecular mechanisms of lipodystrophies and associated insulin resistance and study the relationship between proteins encoded by lipodystrophy genes to understand their function in human adipocyte biology.

* Study the effect of antioxidant micronutrients on the progression of HIV disease in drug abusers on HAART and study the underlying mechanisms of the action of micronutrients on disease progression.

* Support clinical/metabolic studies on the effects of nutritional manipulations to improve body composition and fat redistribution in HIV-infected patients with and without lipodystrophy.

* Study the independent roles of the direct effects of antiretroviral drugs and the secondary effects, such as change in body composition, in metabolic abnormalities in HIV-infected patients; study the role of coinfection with $\mathrm{HCV}$ in metabolic and body composition abnormalities.

* Determine the clinical implications of peripheral fat loss in the context of overweight or obesity, identify women at greatest risk for fracture and determine the efficacy of interventions to prevent further bone loss in this group.

\section{ACKNOWLEDGEMENT}

This study was supported by National Institute on Drug Abuse, Office of Dietary Supplements, National Center for Complementary and Alternative Medicine, National Institutes of Health, U.S. Department of Health and Human Services.

\section{REFERENCES}

1. World Health Organization Report: Neuroscience of Psychoactive Substance Use and Dependence: Summary, 2004, 1211 Geneva 27, Switzerland; telephone: 41-22-7912470; ISBN \# 92-4-159124-2, NLM-WM270.

2. United Nations Office on Drugs and Crime, 2002. Global Illicit Drug Trends 2002, New York, NY. United Nations Office on Drugs and Crime.

3. Substance Abuse and Mental Health Services Administration, 2005. Overview of Findings from the 2004 National Survey on Drug Abuse and Health, Office of Applied Studies, NSDUH Series H-24, DHHS Publications, Rockville, MD.

4. Joint United Nations Programme on HIV/AIDS. AIDS Epidemic Update, 2005. UNAIDS/05.19EWHO/CS/CSR/NCS 2005, English Original, Dec. 2005, Joint United Nations Programme on HIV/AIDS, World Health Organization. 
5. Brown, T.T., A.B. Wisniewski and A.S. Dobs, 2006. Gonadal and adrenal abnormalities in drug users: Cause or consequence of drug use behavior and poor health outcomes. Am. J. Infectious Dis., 2 (3) : 130-135

6. Kumar, M., D. Waldrop-Valverde and A.M. Kumar et al., (2006). Neuroendocrine abnormalities in drug abusers and HIV-infected individuals: Cortisol response to cold pressor challenge. Am. J. Infectious Dis., 2 (3) : 136-140.

7. Tsiodras, S. and C. Mantzoros, 2006. Leptin and Adiponectin in the HIV Associated-Metabolic Syndrome: Physiologic and Therapeutic Implications. Am. J. Infectious Dis., 2 (3): 141-152

8. Kotler, D.P., 2006. The effects of growth hormone therapy in HIV infection. Am. J. Infectious Dis., 2 (3): $153-158$

9. Rao, M.N., G.A. Lee and C. Grunfeld, 2006. Metabolic abnormalities associated with the use of protease inhibitors and non-nucleoside reverse transcriptase inhibitors. Am. J. Infectious Dis., 2 (3): 159-166.
10. Balasubramanyam, A., R.V. Sekhar, F. Jahoor and H.J. Pownall et al., 2006. Pathophysiology of adipocyte defects and dyslipidemia in HIV lipodystrophy: New evidence from metabolic and molecular studies. Am. J. Infectious Dis., 2 (3): 167-172.

11. Baum, M.K., C. Carlin Rafie and S. Lai et al., 2006. Coronary heart disease (CHD) risk factors and metabolic syndrome in HIV-positive drug users in Miami. Am. J. Infectious Dis., 2 (3): 173179.

12. Forrester, J.E., 2006. Nutritional alterations in drug abusers with and without HIV. Am. J. Infectious Dis., 2 (3): 180-183.

13. Grinspoon, S., 2006. Abnormal glucose regulation and treatment strategies for insulin-resistant HIVinfected patients. Am. J. Infectious Dis., 2 (3): 184186.

14. Hruz, P.W., 2006. Molecular mechanisms for altered glucose homeostasis in HIV infection. Am. J. Infectious Dis., 2 (3): 187-192. 